\title{
Isolation, Purification and Structural Identification of Degradation Products from Ketoconazole in a Liquid Formulation: An HPLC and HPLC-MS/MS Study
}

\author{
Fa Zhang, Ph.D* \\ Analytical R \& D, Johnson \& Johnson Consumer Inc, Skillman, USA
}

\begin{abstract}
Ketoconazole is the active ingredient in an antifungal liquid formulation for topical application. A binary gradient HPLC analysis indicated that ketoconazole in the formulation produced four degradation products at minor levels after storage at room temperature for about 3 years. These degradation products were isolated and purified using semi-preparative HPLC procedures followed by analysis using off-line HPLC-ESI-MS and HPLC-ESI-MS/MS. Structures of the degradation products were proposed based on isotope distribution profiles and fragmentation patterns of their molecular ions. Oxidation of ketoconazole appeared to be essential to generate these degradation products. Three of the four degradation products have not been reported in literature.
\end{abstract}

Keywords

Ketoconazole, Antifungal, Oxidation, Degradation, HPLC-MS, HPLC-MS/MS

\section{Introduction}

Ketoconazole (cis-1-acetyl-4-[4-[[2-(2,4-dichlorophenyl)2-(1H-imidazole-1-ylmethyl)-1,3-dioxolan-4-yl]-methoxy] phenyl]piperazine) (I) (Scheme I) is an imidazole antifungal reagent. The activity spectrum of ketoconazole covers a wide variety of yeasts, dermatophytes, and other fungi [1]. Ketoconazole has potential applications in therapy of dermatomycoses, candidiasis of mouth and vagina, systemic candidiasis, chronic mucocutaneous candidiasis and candiduria, as well as deep and subcutaneous mycoses by dimorphic and other fungi [2]. In contrast to other antimycotic drugs of imidazole type, ketoconazole shows good systemic absorption in animals and humans following oral administration of relatively low doses. Ketoconazole can also be administered topically or by intravenous infusion. In addition, there are some reports on efficacy of topical or oral ketoconazole in dermatological disorders not exclusively related to fungi. Ketoconazole has been shown to be highly effective in reducing erythema and hyperkeratosis [3], and even inhibiting lipopolysaccharide induced activation of nitric oxide synthetase gene in murine macrophage cell line J774 [4]. Interestingly, despite extensive applications and studies of ketoconazole, there is quite limited information published regarding degradation chemistry of ketoconazole although there are some scattered reports regarding its stability [5-7]. As for the biotransformation, Whitehouse LW and co-workers [8] investigated metabolites of ketoconazole from liver extract of male Swiss Webster mice; while Miller JD and co-workers [9] studied metabolites of ketoconazole incubated with cryo-preserved human he- patocytes. More than 10 metabolites were identified.

As an anti-fungal reagent, ketoconazole is used as the active ingredient in a liquid formulation which contains ketoconazole at $1 \%$, surfactants, fragrance, colorants, and other ingredients for rinse off topical application. It became necessary to investigate the stability and degradation chemistry of ketoconazole in this formulation to ensure product efficacy and safety. In this report, degradation of ketoconazole in this formulation after storage at room temperature for about three years was studied. Four degradation products (II, III, IV and V) (Scheme I) at minor levels were detected, isolated and purified using reversed-phase high performance liquid chromatographic methods. Their structures were tentatively identified by off-line HPLC electrospray ionization tandem mass spectrometry (HPLC-ESI-MS and HPLC-ESI-MS/MS). Three of the four degradation products, i.e. III, IV and V, have not been reported in literature.

* Corresponding author: Fa Zhang, Ph.D, Research Director, Analytical R \& D, Johnson \& Johnson Consumer Inc, Skillman, NJ 08558, USA, Tel: (908)-874-1333

Accepted: July 04, 2018

Published online: July 06, 2018

Citation: Zhang F (2019) Isolation, Purification and Structural Identification of Degradation Products from Ketoconazole in a Liquid Formulation: An HPLC and HPLC-MS/MS Study. Chromatogr Spectrosc Tech 2(1):12-20 

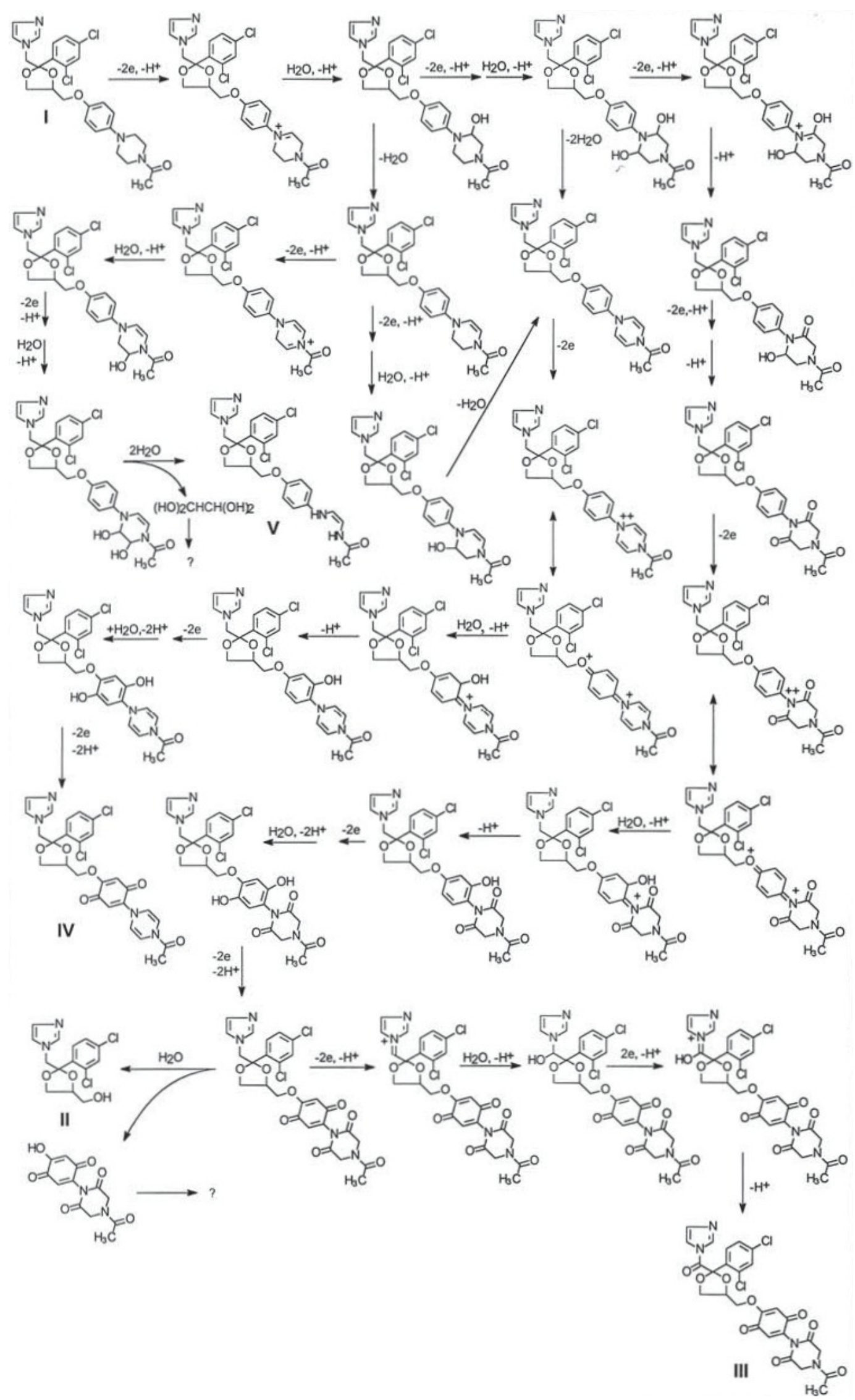

Scheme I: Imidazole antifungal reagent. 
Citation: Zhang F (2019) Isolation, Purification and Structural Identification of Degradation Products from Ketoconazole in a Liquid Formulation: An HPLC and HPLC-MS/MS Study. Chromatogr Spectrosc Tech 2(1):12-20

\section{Experimental}

\section{Material and reagents}

Ketoconazole used was a USP reference standard. 2-(2,4-dichlorophenyl)-2-(1H-imidazol-1-ylmethyl)-1,3-dioxalan-4-methanol (II) was obtained from Janssen Biotech through Research Diagnostics, Inc., Pleasant Hill Rd., Flanders, NJ 07836. HPLC grade methanol, acetonitrile and chloroform were obtained from Fisher Scientific. Ammonium formate, methylene chloride, phosphoric acid and ammonium hydroxide were obtained from J. T. Baker. HPLC grade water was prepared using a Modulab Polisher I HPLC Laboratory Reagent Grade Water System (Continental Water System Corp., Bensalem, PA).

\section{Sample extraction}

$14 \mathrm{~g}$ of $1 \%$ ketoconazole liquid formulation stored for about three years at room temperature was suspended in 3 $\mathrm{mL}$ of methanol and $20 \mathrm{~mL}$ of methylene chloride. After stirring at room temperature for 0.5 hours, the mixture was kept at room temperature overnight to produce distinguishable organic and aqueous phases. The organic layer was isolated and evaporated by purging compressed nitrogen (10-20 psi, $\sim 2$ hours). The residue was subsequently suspended in $5 \mathrm{~mL}$ of methanol and kept at $4{ }^{\circ} \mathrm{C}$ for more than 1 hour. The undissolved solid was removed by filtration (Autovial Syringeless Filter, PTFE, $0.45 \mu \mathrm{m}$, Whatman) and the filtrate was used for HPLC isolation of the degradation products.

\section{HPLC conditions}

Two semi-preparative reversed-phase HPLC methods (HPLC I and HPLC II) and one analytical reversed-phase HPLC method (HPLC III) were developed. HPLC I was used for preliminary isolation of the degradation products. HPLC II was used for subsequent purification of the degradation products. HPLC III, as an analytical method, was used for analysis of the isolated degradation products and the liquid formulation as well as placebo. The liquid formulation and placebo were suspended into methanol and filtrated prior to injection into HPLC system for analysis. HPLC I and HPLC II used a Ranin Rabbit HPLC system equipped with two HPX pumps, a Rheodyne injector, a Knauer variable wavelength monitor at $230 \mathrm{~nm}$, a Waters GCM column oven at $40{ }^{\circ} \mathrm{C}$, and a Develosil UG-3 ODS column $(3 \mu \mathrm{m}, 10 \times 0.6 \mathrm{~cm})$ with a Develosil UG-5 $(1 \times$ $0.4 \mathrm{~cm}$ ) guard cartridge. For HPLC III, a HP1090L HPLC with detection at $230 \mathrm{~nm}$ with Agilent ChemStation for instrument control and diode array detection. Four HPLC mobile phases were used. Mobile phase A was prepared by adding $18.4 \mathrm{~g}$ of phosphoric acid (85\%) into $4 \mathrm{~L}$ of deionized water and the $\mathrm{pH}$ was then adjusted to 9.0 by adding ammonium hydroxide (29.6\%). Mobile phase B was prepared by mixing $3600 \mathrm{~mL}$ of methanol with $400 \mathrm{~mL}$ of mobile phase $A$. Mobile phase $C$ was prepared by adding $3 \mathrm{~mL}$ of formic acid into $4 \mathrm{~L}$ of deionized water and then $\mathrm{pH}$ was adjusted to 4.0 by adding ammonium hydroxide. Mobile phase D was prepared by mixing $900 \mathrm{~mL}$ of methanol with $100 \mathrm{~mL}$ of mobile phase C. For HPLC I, the following binary gradient was used with mobile phases $A$ and B: 0-1 min., 50\% mobile phase A, 1-15 min., linear gradient to
$61.6 \%$ mobile phase $B, 15-45$ min., linear gradient to $66.7 \%$ mobile phase $B, 45-50$ min., linear gradient to $100 \%$ mobile phase B. Flow rate was set as $1.7 \mathrm{~mL} / \mathrm{min}$. For HPLC II, the following binary gradient was used with mobile phases $C$ and D: 0-1 min., 39\% mobile phase D, 1-35 min., linear gradient to $60.6 \%$ mobile phase D, 35-40 min., linear gradient to $100 \%$ mobile phase D. Flow rate was $1.7 \mathrm{~mL} / \mathrm{min}$. For HPLC III, the following binary gradient was used with mobile phase $A$ and methanol: 0-1 min., 45\% methanol, 1-15 min., linear gradient to $55 \%$ methanol, $15-45 \mathrm{~min}$., linear gradient to $60 \%$ methanol, $45-50$ min., linear gradient to $90 \%$ methanol. Flow rate was $1 \mathrm{ml} / \mathrm{min}$.

\section{Off-line HPLC-MS}

Mass spectrometric analysis was performed using a Finnigan MAT TSQ 700 triple quadrupole mass spectrometer with an ESI source (San Jose, CA, USA). The spray voltage was set to $4.5 \mathrm{kV}$ and the heated capillary temperature was maintained at $200{ }^{\circ} \mathrm{C}$. The instrument was optimized in the positive ion mode. For tandem spectrometric measurement, argon was used as the collision gas at a pressure of $2.5 \mathrm{~m}$ Torr, and the collision energy was maintained at $-40 \mathrm{eV}$.

The HPLC procedure for introducing samples into mass spectrometric detector used a narrow bore column (YMC BASIC, $250 \times 2.0 \mathrm{~mm}, 5 \mu \mathrm{m}$ particle size) and Waters Model $600 \mathrm{E}$ pump operated at $0.25 \mathrm{~mL} / \mathrm{min}$ using mobile phases $\mathrm{E}$ and $F$. Mobile phase $E$ was a solution of $5 \%$ acetonitrile, $1 \%$ methanol with $5 \mathrm{mM}$ ammonium formate adjusted to $\mathrm{pH}$ 6.2. Mobile phase $F$ was a solution of $45 \%$ acetonitrile, $1 \%$ methanol with $5 \mathrm{mM}$ ammonium formate adjusted to $\mathrm{pH}$ 6.2. The elution used a binary gradient with $80 \%$ of mobile phase E for 3 min initially and then linearly changed to $100 \%$ mobile phase $\mathrm{F}$ at $25 \mathrm{~min}$.

\section{Results}

\section{Chromatographic analysis of the liquid formula- tion and isolation of the degradation products}

HPLC chromatograms of the liquid formulation after stored for about 3 years at room temperature along with corresponding placebo are presented in Figure 1 using method HPLC III. The placebo has the same ingredients as the formulation but without ketoconazole. The liquid formulation and placebo were diluted using methanol and filtered prior to injection into HPLC for analysis. Peak I is due to ketoconazole and at least four suspected degradation products were observed, which were labeled as II, III, IV and V, respectively. Peak II was confirmed to be 2-(2,4-dichlorophenyl)-2-(1H-imidazol-1-ylmethyl)-1,3-dioxalan-4-methanol by comparing its identical HPLC retention time and online UV spectrum with that of an authentic compound under identical chromatographic conditions. The levels of II, III, IV and V were determined to be $0.08 \%, 0.18 \%, 0.45 \%$ and $0.29 \%$, respectively, normalized with ketoconazole HPLC peak area\%. Figure 1 also indicated that there is a peak at about 17 minute which is larger in the aged formulation than in the placebo; this peak co-eluted with the ingredients of placebo and remains to be studied. In this report, only degradation products II, III, V and 


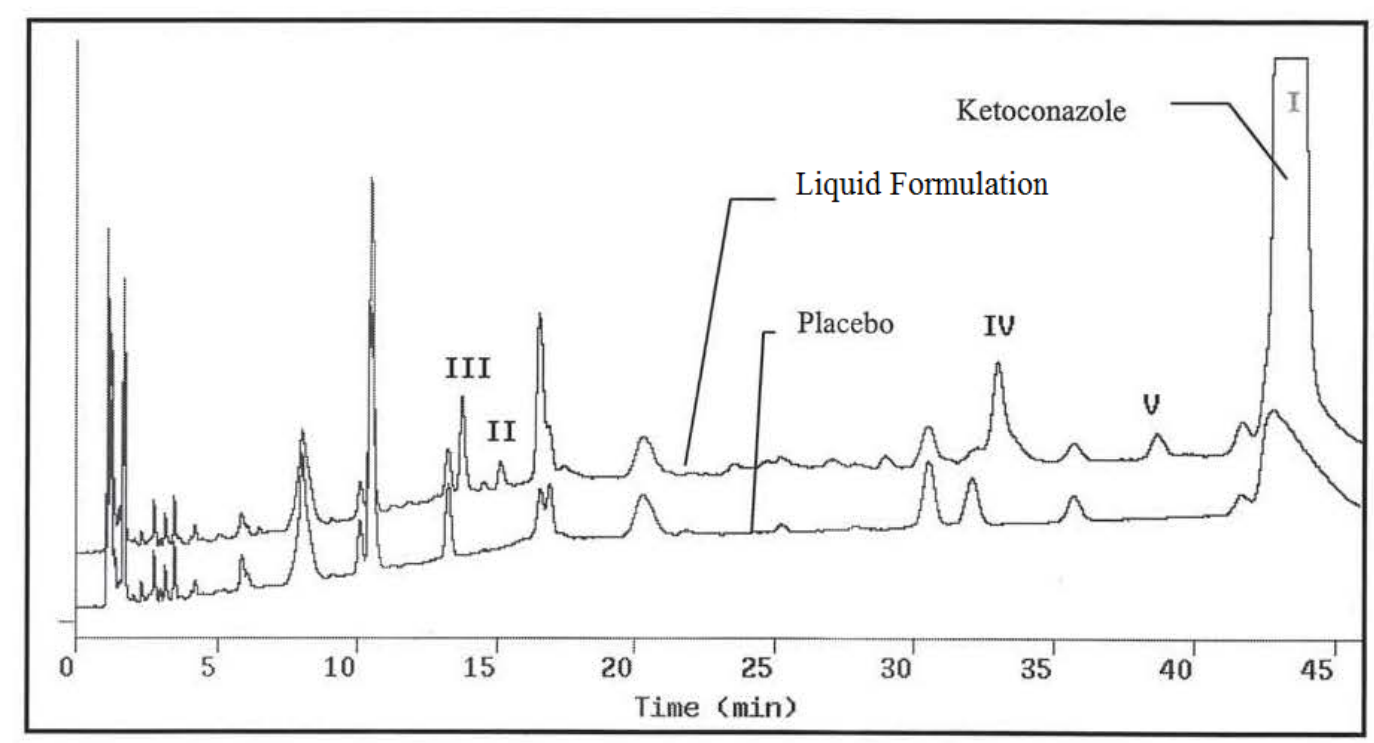

Figure 1: HPLC Chromatograms of Aged Liquid Formulation and Placebo.

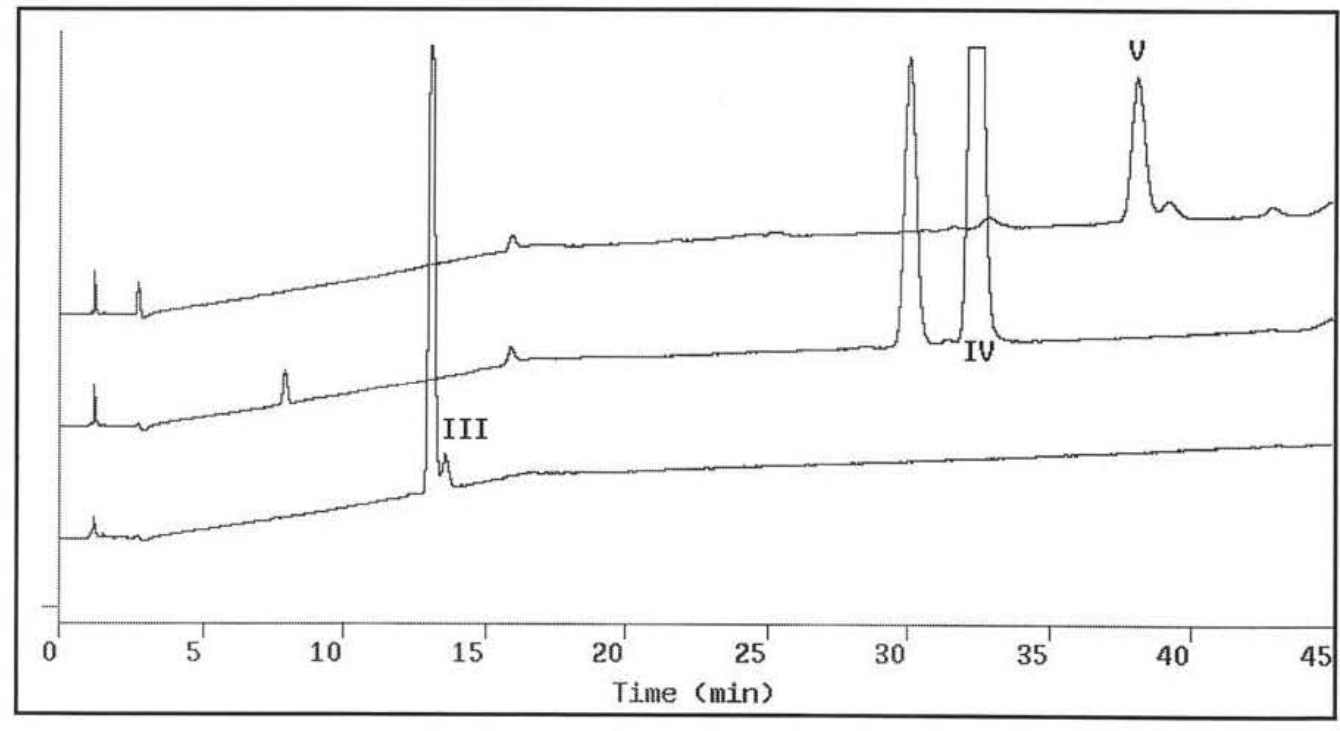

Figure 2: HPLC Chromatograms of III', IV', and V'.

$\mathrm{V}$ were investigated. It was also found that the levels of II, III, IV and V increased with increase in storage time. None of the degradation products were observed when the formulation was freshly manufactured. After storage for 18 months which is the shelf life of the product the levels of degradation products increased but none of them were beyond $0.1 \%$. The degradation product formation seemed to be accelerated after 18-month storage even none of the excipients was likely capable of interacting with ketoconazole chemically.

Due to the complicated formulation excipients resulting in interference and ion suppression obtaining decent HPLC-MS spectra of the degradation products became challenging. An approach of chromatographic isolation/purification followed by off-line HPLC-MS analysis was performed. To isolate degradation products III, IV and V from the aged liquid formulation,
0.2-0.5 $\mathrm{mL}$ aliquots of the formulation extract, prepared according to the procedure described in the experimental section of this report, were first chromatographed using HPLC I. The eluents at 12.4, 28.4 and $32.9 \mathrm{~min}$. were collected individually and labeled as III', IV' and V', respectively. Figure 2 presents the analytical chromatograms of III', IV', and V' using HPLC III. A large amount of unwanted material had been removed and the number of chromatographic peaks decreased from more than 16 to less than four for each of the degradation product fractions. For further purification, $0.5-2 \mathrm{~mL}$ of III', or IV', or V' solutions were injected and eluted using method HPLC II. The eluents at 27.4, 29.7, and 33 min were individually collected and labeled as III, IV and V. Figure 3 shows the analytical chromatograms of III, IV and V to exhibit single peaks indicating pure degradation products obtained. Peak purity measurements using HP ChemStation indicated that peaks III, 


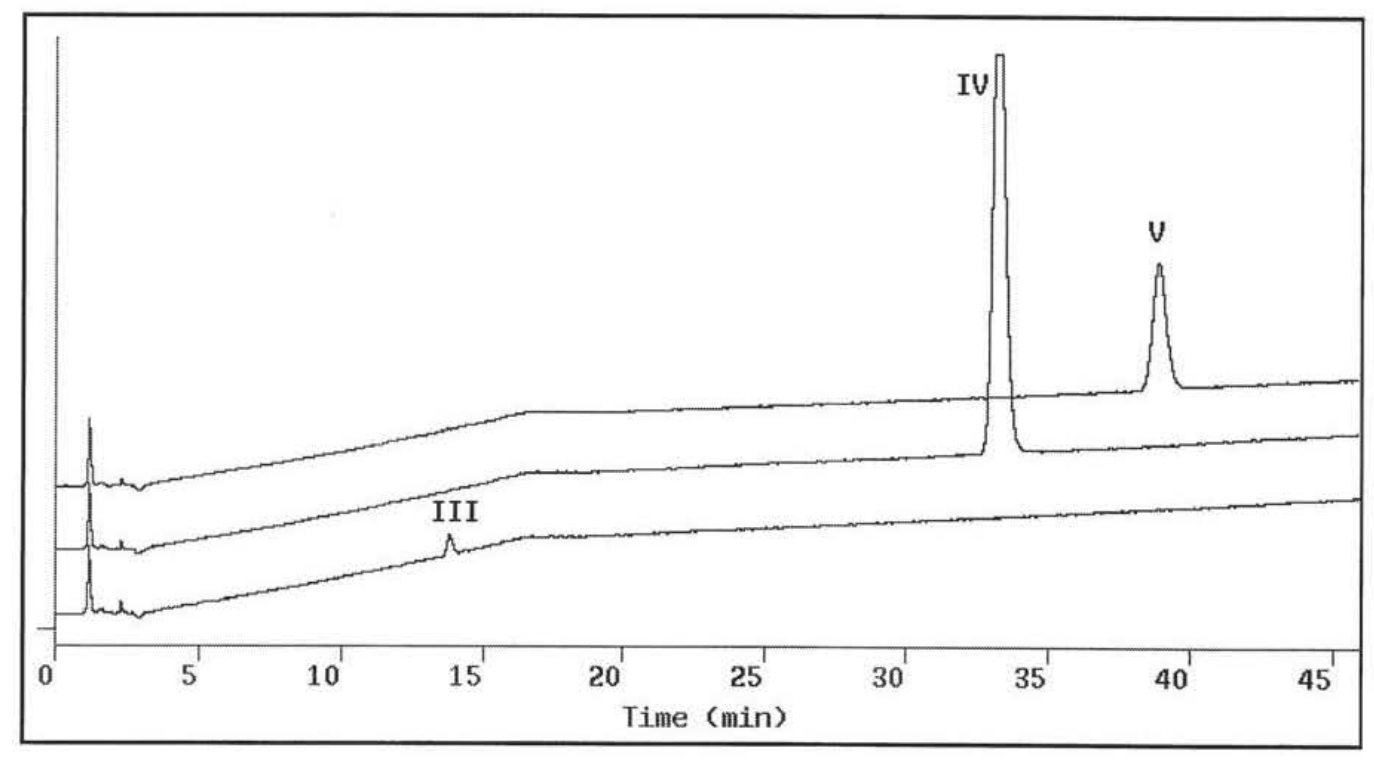

Figure 3: Chromatograms of III, IV and V.

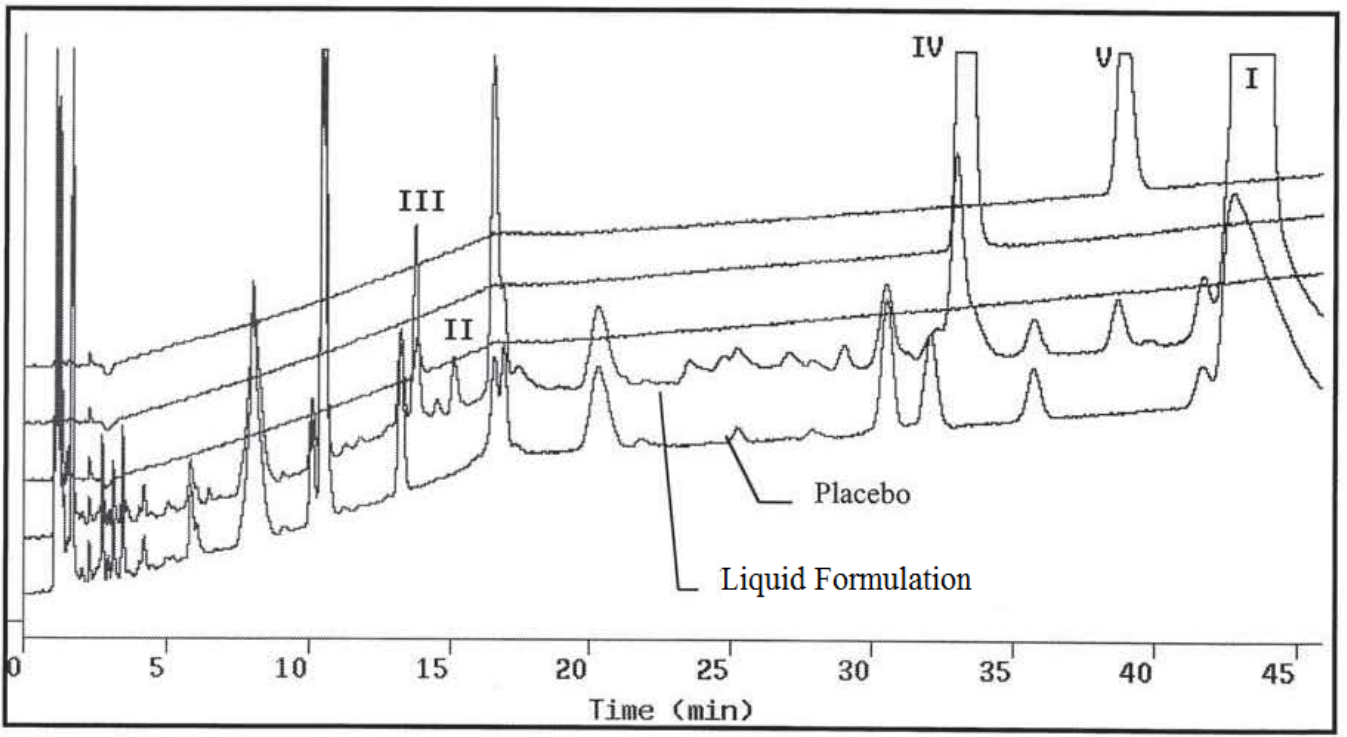

Figure 4: HPLC Chromatograms of III, IV, V, Aged Liquid Formulation, and Placebo.

IV and $V$ were homogenous. The above isolation and purification sequence was repeated until adequate amounts of pure degradation products were obtained for following HPLC-MS studies.

Figure 4 shows comparison of the analytical chromatograms of aged formulation, placebo, III, IV and V.

\section{HPLC-MS studies of III, IV and V}

The isolated degradants III, IV and V were analyzed offline using HPLC-ESI-MS in full scan mode and HPLC-ESI-MS/ MS on identified molecular ions. The full scan mass spectra of III, IV and V are presented in Figure 5a, Figure 6a and Figure 7a.

Their mass spectra displayed characteristic isotope clus- ter patterns due to the chlorine atoms and their molecular weights were determined as 602, 556 and 502 for III, IV and $\checkmark$ with molecular compositions of $\mathrm{C}_{26} \mathrm{H}_{20} \mathrm{~N}_{4} \mathrm{O}_{9} \mathrm{Cl}_{2}, \mathrm{C}_{26} \mathrm{H}_{22} \mathrm{~N}_{4} \mathrm{O}_{6} \mathrm{Cl}_{2}$ and $\mathrm{C}_{24} \mathrm{H}_{24} \mathrm{~N}_{4} \mathrm{O}_{4} \mathrm{Cl}_{2}$, respectively. The isotope distribution profiles of the protonated molecular ions are consistent with the theoretical isotope distribution profiles. In order to obtain additional structural information, the fragmentation patterns of the molecular ions of III, IV and V were investigated using $\mathrm{HPLC}-\mathrm{MS} / \mathrm{MS}$. Figure $5 \mathrm{~b}$, Figure $6 \mathrm{~b}$ and Figure $7 \mathrm{~b}$ present the observed diagnostic fragments from the corresponding molecular ions along with the proposed structures of III, IV and $\mathrm{V}$. The proposed structures are tentative and were based only on HPLC-MS and HPLC-ESI-MS/MS studies. Due do the complexity of the formulation matrix and the minor levels of the degradants formed plus limited amount of the liquid formulation sample, it was not possible to obtain adequate amounts 
Citation: Zhang F (2019) Isolation, Purification and Structural Identification of Degradation Products from Ketoconazole in a Liquid Formulation: An HPLC and HPLC-MS/MS Study. Chromatogr Spectrosc Tech 2(1):12-20

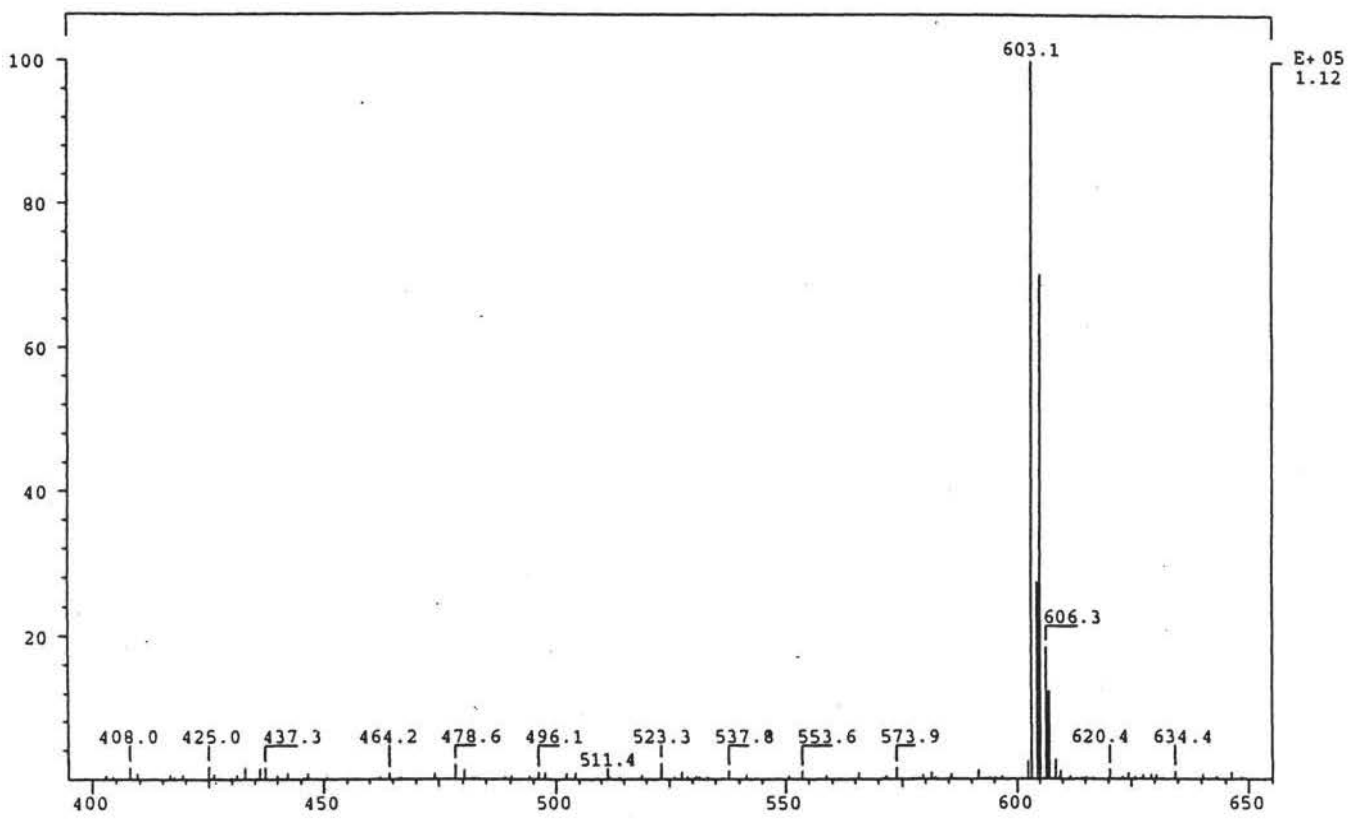

Figure 5a: Full Scan Mass Spectrum of III.
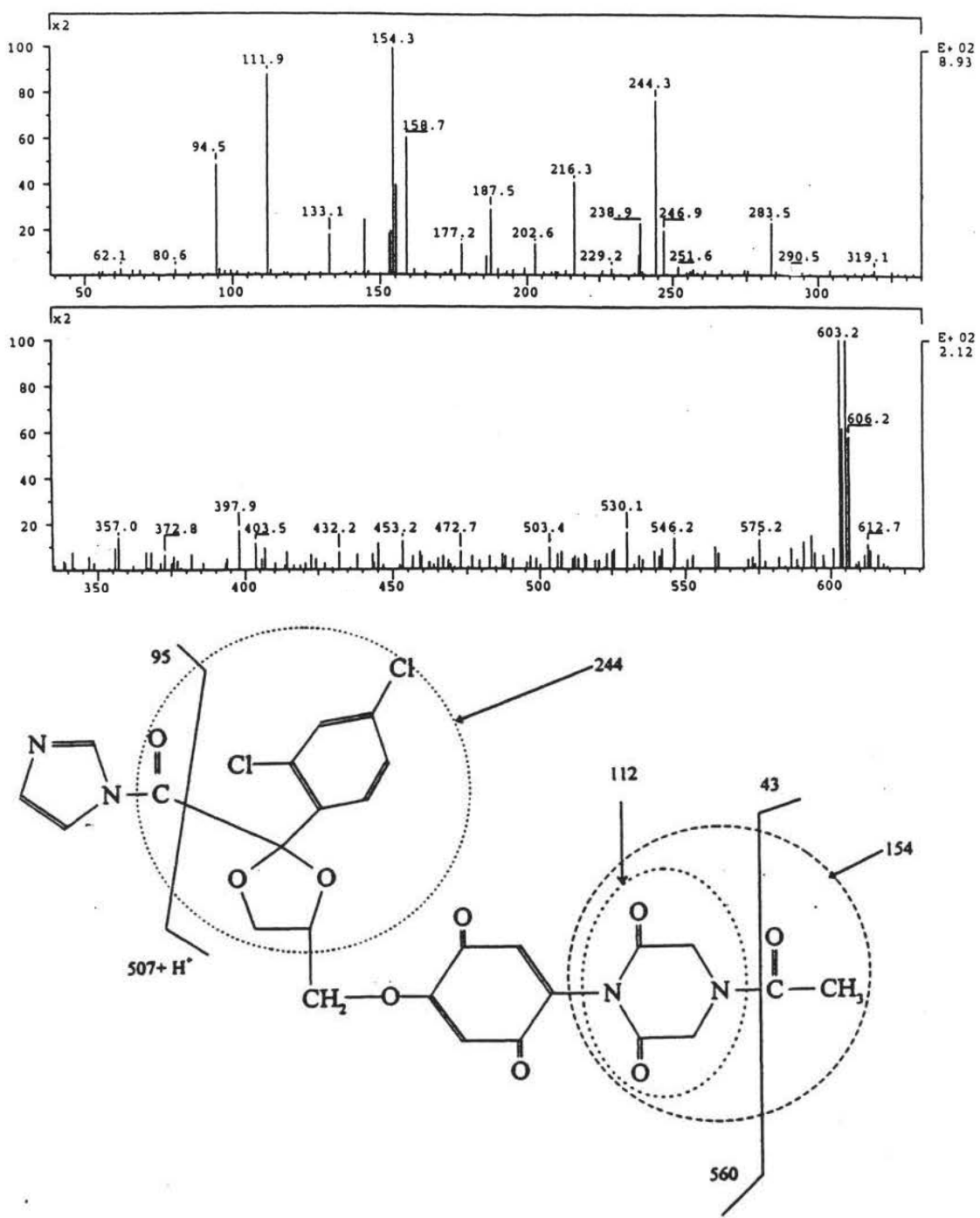

Figure 5b: MS/MS Diagnostic Fragments and Proposed Structure of III. 


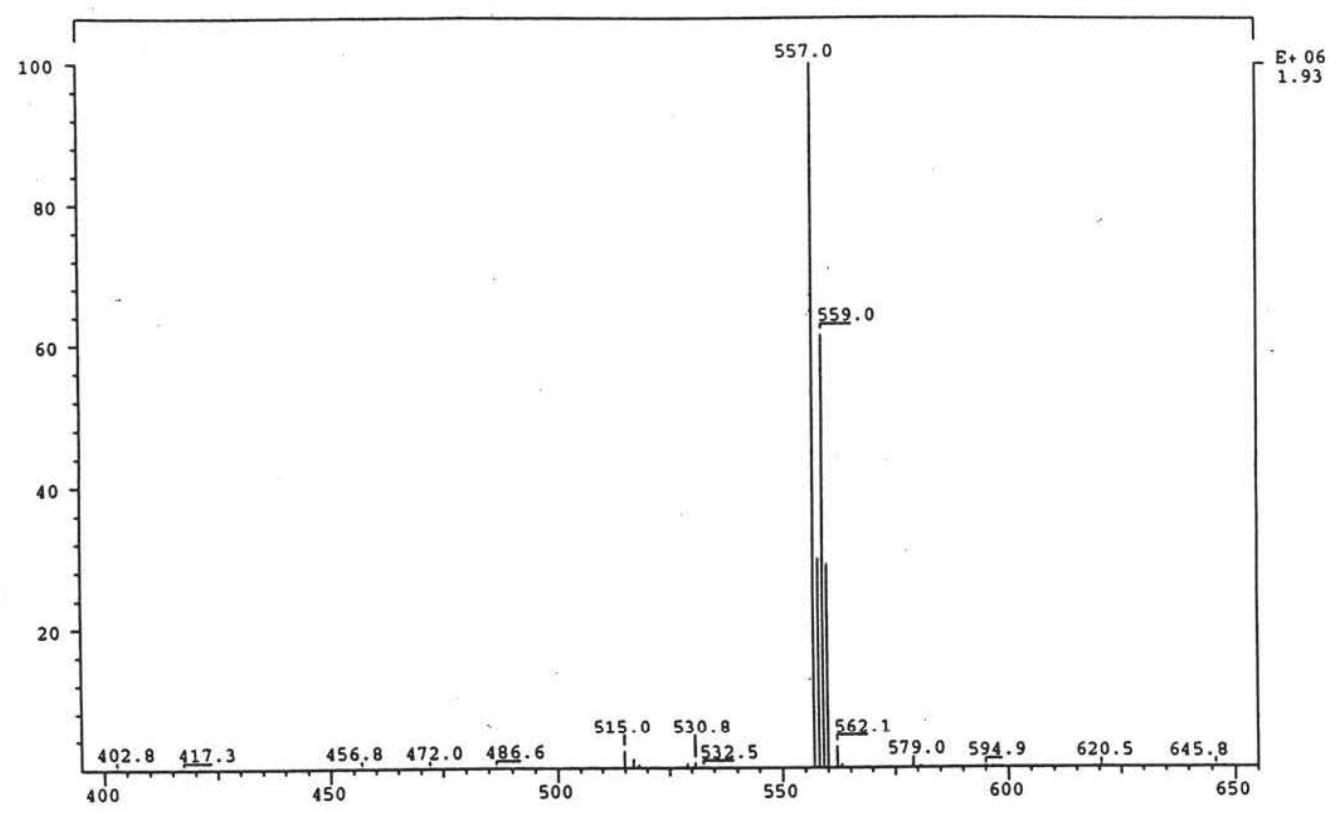

Figure 6a: Full Scan Mass Spectrum of IV.
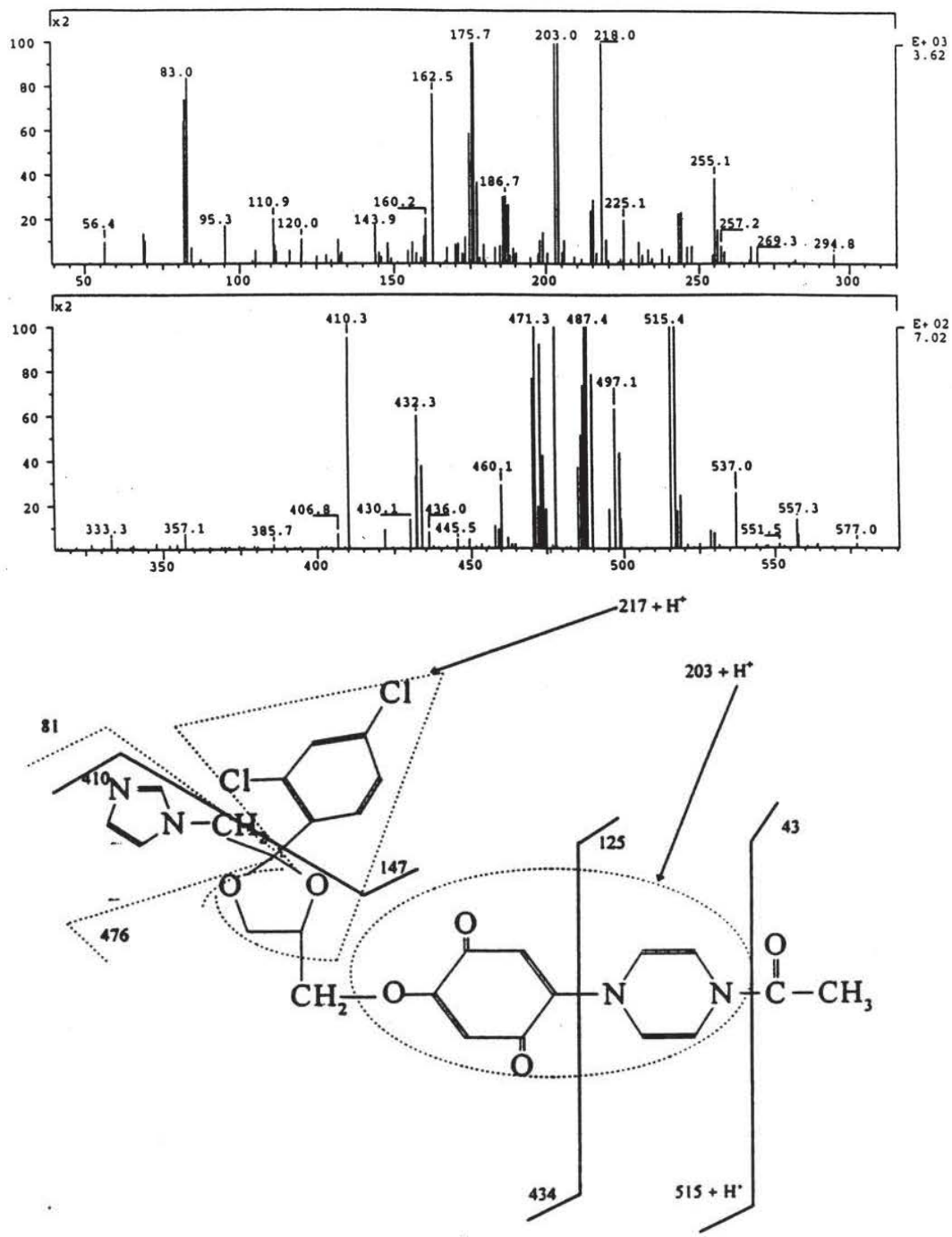

Figure 6b: MS/MS Diagnostic Fragments and Proposed Structure of IV. 
Citation: Zhang F (2019) Isolation, Purification and Structural Identification of Degradation Products from Ketoconazole in a Liquid Formulation: An HPLC and HPLC-MS/MS Study. Chromatogr Spectrosc Tech 2(1):12-20

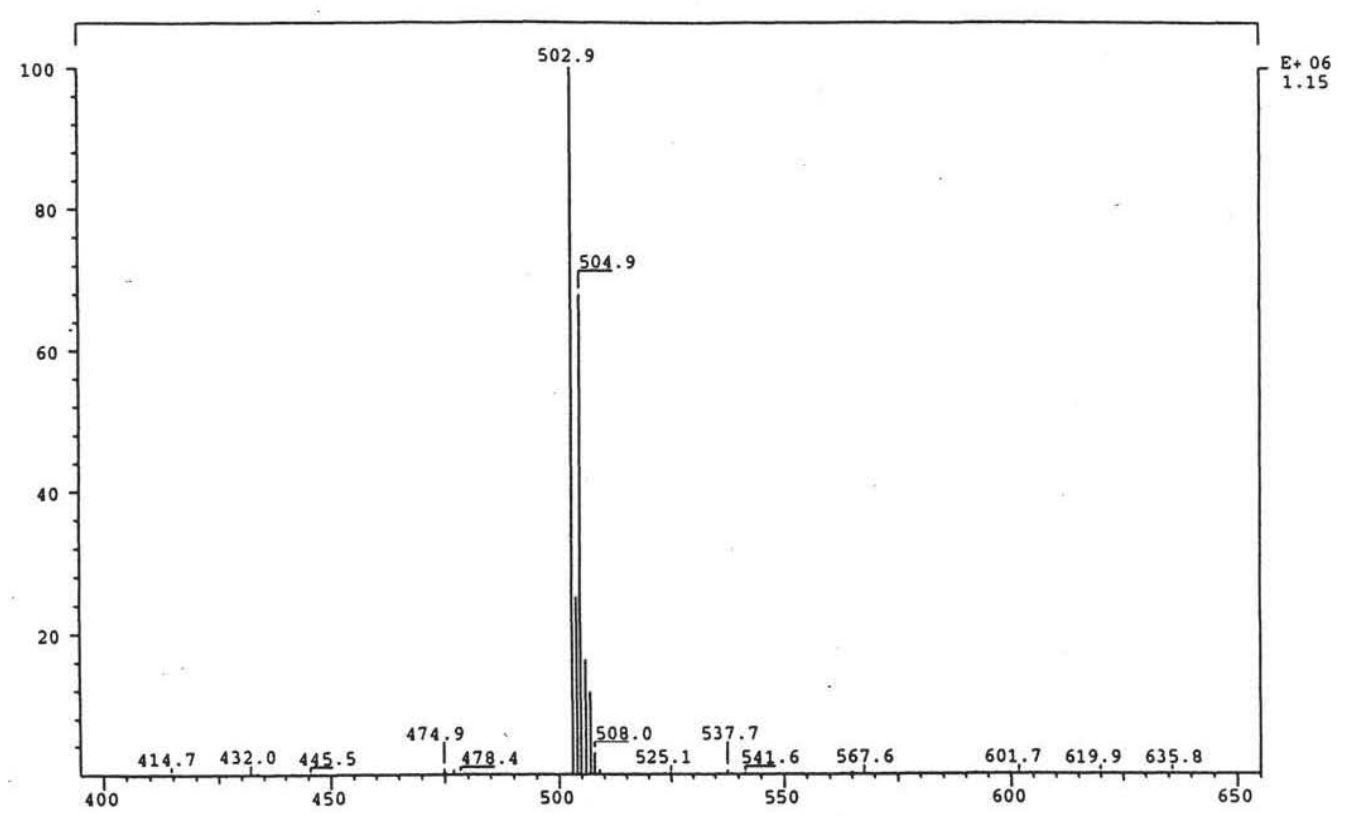

Figure 7a: Full Scan Mass Spectrum of V.
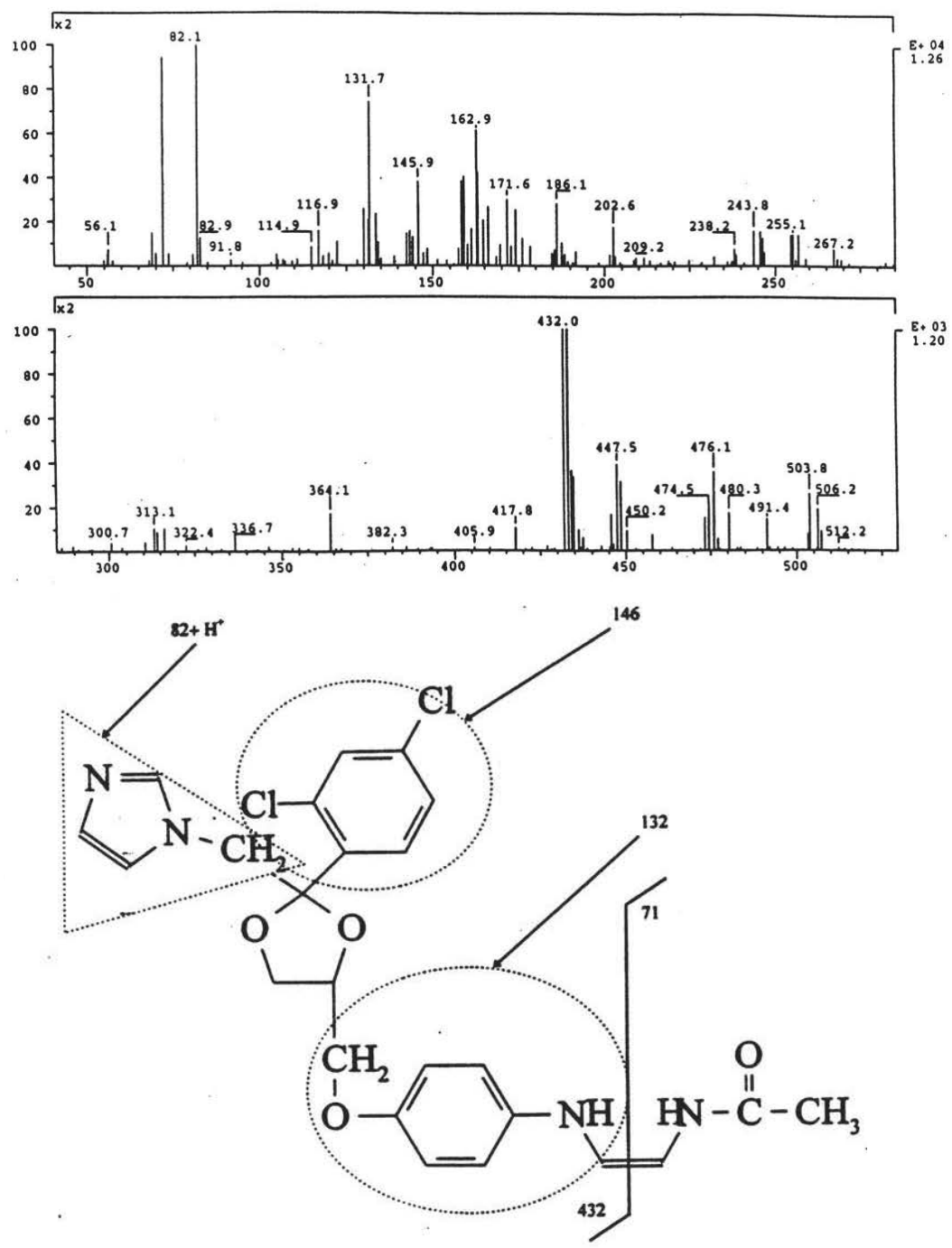

Figure 7b: MS/MS Diagnostic Fragments and Proposed Structure of V. 
of the pure degradation products for additional structural investigation, such as NMR studies.

\section{Discussion}

In this report, four degradation products at minor levels from ketoconazole (I) in a liquid formulation sample after storage at room temperature for about 3 years were isolated using semipreparative HPLC and tentatively identified using HPLC-ESI-MS and HPLC-ESI-MS/MS analyses. Three of the four degradation products have not been reported in literature. Based on the comparison between the structures of ketoconazole and that of degradation products II, III, IV, and $\mathrm{V}$, it is concluded that II might be formed from hydrolysis of ketoconazole. However, to generate III, IV and V, likely a series of oxidation, hydroxylation and O-dealkylation need to occur. Oxidation of ketoconazole must be essential. The substituted aromatic amine nucleus of ketoconazole has a pair of free electrons to make it susceptible to oxidation. As a matter of fact, oxidation, cleavage of the imidazole and piperazine rings, O-dealkylation, and hydroxylation were reactions observed in the biotransformation of ketoconazole to yield various metabolites $[8,9]$ although structures of the reported metabolites are different from II, III, IV and V. Besides directly hydrolysis of ketoconazole degradation product II might also be generated from hydrolysis of oxidized ketoconazole since its formation was accelerated after long time storage. A proposed potential mechanism for the formation of the degradation products are presented in Scheme I. No safety evaluation was performed on the degradation products since within 18 month shelf life the degradation product levels were less than $0.1 \%$ and the liquid formulation is for topical rinse off application with low systemic absorption.

\section{Acknowledgement}

Dr. Tarit K. Mukherjee collected the mass spectrometric spectra.

\section{References}

1. Levine HB (1982) Ketoconazole in the Management of Fungal Disease. ADIS press, Australia.

2. Heeres J, Backx LJ, Van Cutsem J (1984) Antimycotic azoles. 7. Synthesis and antifungal properties of a series of novel triazolones. J Med Chem 27: 894-900.

3. Van Cutsem J (1989) Animal models for dermatomycotic infections. Curr Top Med Mycol 3: 1-35.

4. Baroni A, Ruocco V, De Paolis P, et al. (1999) Ketoconazole inhibits lipopolysaccharide induced activation of the nitric oxide synthase gene in the murine macrophage cell line J774. Arch Dermatol Res 291: 54-58.

5. Allen LV Jr, Erickson MA 3rd (1996) Stability of ketoconazole, metolazone, metronidazole, procainamide hydrochloride, and spironolactone in extemporaneously compounded oral liquids. Am J Health Syst Pharm 53: 2073-2078.

6. Kumer KP, Okonomah AD, Bradshaw WG, et al. (1991) Stability of ketoconazole in ethanolic solution. Drug Dev Ind Pharm 17: 577-580.

7. Lee Robin LH (1988) Stability of oily ketoconazole eye drops on dry heating. Aust J Hosp Pharm 18: 117-119.

8. Whitehouse LW, Menzies A, Dawson B, et al. (1994) Mouse hepatic metabolites of ketoconazole: Isolation and structure elucidation. J Pharm Biomed Anal 12: 1425-1441.

9. Miller JD, Rodriguez RJ, Chang SY, et al. (2000) Characterization of N-Deacetylated ketoconazole metabolites produced by a Recombinant Human FMO System using Triple Quadrupole and QqTOF Instruments. The $48^{\text {th }}$ ASMS Conference, Long Beach, CA, USA.

DOI: $10.36959 / 326 / 767$

Copyright: (C) 2019 Zhang F. This is an open-access article distributed under the terms of the Creative Commons Attribution License, which permits unrestricted use, distribution, and reproduction in any medium, provided the original author and source are credited. 